\title{
Sono, estado nutricional e hábitos de vida de caminhoneiros
}

\author{
Sleep, nutritional status and life habits of truckers
}

Patrícia De Paris ${ }^{1}$, Greice Grandi², Josiane Siviero ${ }^{3}$, Fernanda Bissigo Pereira ${ }^{4}$

\section{RESUMO}

Objetivo: Avaliar o período de sono, o estado nutricional e os hábitos de vida dos caminhoneiros do município de Veranópolis/RS.

Materiais e Métodos: Estudo retrospectivo, transversal com dados secundários de uma amostra de conveniência de 100 caminhoneiros entre 21 e 60 anos. Estudou-se variáveis sociodemográficas, de hábitos de vida, relato de doença crônica não transmissível (DCNT), uso de medicamentos para dormir ou para tratamento de saúde, questões sobre as práticas alimentares e parâmetros antropométricos. Os dados foram analisados por estatística descritiva e analítica pelo programa SPSS® com nível de significância de $5 \%$.

Resultados: A idade média dos caminhoneiros foi 38,5 $\pm 10,2$ anos. A maioria dorme 6,0 $\pm 1,4$ horas/dia, apresenta-se com sobrepeso e obesidade (82\%) e risco para o desenvolvimento de doenças cardiovasculares (69\%). O tempo de sono teve associação com o colesterol total e o diabete mellitus. A média de refeições realizadas por dia foi de $3,4 \pm 0,9$, prevalecendo o café da manhã, o almoço e o jantar. Caminhoneiros que dormem a semana inteira em casa realizam o café da manhã, o almoço e o jantar em suas residências, enquanto aqueles que dormem de 1 a 5 dias fora de casa realizam o almoço e o jantar em restaurantes. Houve associação significativa da faixa etária $(p=0,044)$, consumo de bebidas alcoólicas $(p=0,020)$ e realização de lanche da tarde $(p=0,013)$ com dormir após o almoço; e dos obesos com a presença de hipertensão $(p=0,035)$.

Conclusão: O sobrepeso e a obesidade caracterizaram o estado nutricional dos caminhoneiros. O sono associou-se com as DCNT. A maioria realiza três refeições diárias, não pratica exercícios físicos e não é tabagista, porém, tem o hábito de ingerir bebidas alcoólicas.

Palavras-chave: estado nutricional; sono; comportamento alimentar.

\begin{abstract}
Objective: To evaluate the sleep period, the nutritional state and the lifestyle habits of truck drivers from Veranópolis/RS. Materials and Methods: The study is cross-sectional, retrospective, with secondary data from a convenience sample of 100 truck drivers between 21 and 60 years old. Socio-demographic variables, lifestyle habits, history of chronic noncommunicable disease (CNCD), use of sleeping medication or other treatment of health issues, questions about dietary and anthropometric parameters were studied. The data were analyzed using descriptive and analytical statistics through the program SPSS with a significance level of $5 \%$.

Results: The average age of truck drivers was $38.5 \pm 10.2$ years old. Most of them sleep $6.0 \pm 1.4$ hours/day, present overweight and obesity (82\%) and risk for developing cardiovascular disease (69\%). The sleep period was associated with total cholesterol and diabetes mellitus. The average number of meals eaten per day was $3.4 \pm 0.9$, prevailing breakfast, lunch and dinner. Truck drivers who sleep the whole week at home usually have breakfast, lunch and dinner at their homes, while those who sleep 1-5 days away from home have lunch and dinner in restaurants. There was a significant association between age $(p=0.044)$, alcohol consumption $(p=0.020)$ and afternoon snack $(p=0.013)$ with sleep after lunch, and between obesity and hypertension ( $p=0.035)$.

Conclusions: Overweight and obesity characterized the nutritional status of the truck drivers. The sleep was associated with CNCDs. The biggest part of the truck drivers has three daily meals, does not practice exercises and does not smoke, however, has the habit of drinking alcohol.
\end{abstract}

Keywords: nutritional state; sleep; feeding behavior.

\footnotetext{
${ }^{1}$ Acadêmica do Curso de Graduação em Nutrição da Faculdade Cenecista de Bento Gonçalves/RS (CNEC).

${ }^{2}$ Nutricionista graduada pela CNEC.

${ }^{3}$ Nutricionista. Doutora em Ciências da Saúde pela Pontifícia Universidade Católica do Rio Grande do Sul (PUCRS). Docente da CNEC e do Curso de Nutrição da Universidade de Caxias do Sul/RS (UCS).

${ }^{4}$ Nutricionista. Mestre em Inclusão Social e Acessibilidade pela Universidade Feevale (RS). Docente da Faculdade da Serra Gaúcha (FSG) de Caxias do Sul/RS.
} 


\section{INTRODUÇÃO}

O trabalho do ser humano deve ser uma prática livre e autônoma, contribuindo para seu crescimento e evolução, suprindo suas necessidades e carências. No processo de trabalho ocorrem mudanças. Inicialmente era visto como uma atividade sofrida, desgastante e, nos dias atuais, seu conceito consiste em direito a ser conquistado. A categoria profissional a ser estudada é a do motorista de caminhão, pois estes são de grande importância para a movimentação da economia no país ${ }^{1}$. Segundo o Ministério do Trabalho e Emprego $(\mathrm{MTE})^{2}$, "motoristas de caminhão são trabalhadores autônomos ou assalariados que prestam serviço para empresas do ramo de logística ou transporte terrestre". Esses trabalhadores sofrem influência de alguns fatores relacionados às condições de saúde, trabalho e estilo de vida em decorrência do exercício da sua atividade profissional. O horário de trabalho é um ponto a ser avaliado, pois muitos motoristas trabalham em horários irregulares, e acabam adotando hábitos alimentares não saudáveis como "beliscar", principalmente alimentos que constituem fontes de lipídeos, bebidas cafeinadas e álcool, mais frequentemente no período noturno. Os restaurantes de beira de estrada, onde os motoristas costumam fazer suas refeições diariamente, nem sempre oferecem preparações nutritivas. Na maioria das vezes, elas são de alto valor calórico ${ }^{3}$.

Além disso, é bastante comum entre os motoristas o uso de álcool e anfetaminas para redução do sono e do cansaço, pois muitos precisam cumprir horários e, para isso, recorrem ao uso dessas drogas ${ }^{4}$. Tem-se observado que o curto tempo de sono tem relação com o aumento do Índice de Massa Corporal (IMC), ou seja, motoristas que dormem menos de seis horas por dia aumentam o risco de obesidade. As alterações nos horários do sono influenciam também o apetite, a saciedade e a quantidade de ingestão de alimentos, favorecendo, assim, a obesidade e as doenças crônicas não transmissíveis (DCNT) ${ }^{5}$.

As extensas jornadas de trabalho dos motoristas de caminhão contribuem para o sedentarismo. Ao dirigir, o gasto energético é baixo devido à inexistência de práticas de atividade física regular, e isso aumenta os danos à saúde, com o aparecimento de diabetes, obesidade, hipertensão, estresse, depressão, entre outras patologias ${ }^{6}$. Com base nessas considerações, o presente estudo teve como objetivo avaliar o período de sono, o estado nutricional e os hábitos de vida dos caminhoneiros do município de Veranópolis - RS.

\section{MATERIAIS E MÉTODOS}

Estudo descritivo retrospectivo de delineamento transversal, no qual foram utilizados dados secundários provenientes de um banco de dados constituído por uma amostra de conveniência de 100 caminhoneiros do município de Veranópolis/RS. Foram incluídos no estudo somente os caminhoneiros do sexo masculino entre 21 e 60 anos de idade, residentes no município de Veranópolis/RS, e que consentiram sua participação no estudo após assinatura do Termo de Consentimento Livre e Esclarecido (TCLE). Para obtenção dos dados, os caminhoneiros foram abordados em empresas, postos de combustíveis, borracharias e oficinas mecânicas da cidade.

Fizeram parte das variáveis estudadas na pesquisa os dados referentes aos parâmetros sociodemográficos (renda e escolaridade), hábitos de vida (período de sono, atividade física, tabagismo e etilismo), relato de DCNT (hipertensão arterial, diabetes, colesterol e triglicerídeos elevados), uso de medicamentos para dormir ou para não dormir, questões sobre as práticas alimentares (número, horário e local das refeições/dia) e parâmetros antropométricos (IMC e circunferência abdominal).

A medida da estatura foi aferida com uma fita métrica fixada verticalmente em uma parede, de modo a fazer um ângulo de $90^{\circ} \mathrm{com}$ o piso. O peso foi verificado em uma balança portátil digital (Philco® PHBE10) com capacidade para $150 \mathrm{Kg}$, previamente aferida. As medidas de peso e estatura foram realizadas com o indivíduo descalço e usando roupas leves. O IMC foi determinado por meio do cálculo da razão entre a medida do peso em quilogramas dividido pela estatura em metros elevado ao quadrado: peso $(\mathrm{Kg}) /$ estatura $\left(\mathrm{m}^{2}\right)$. O estado nutricional foi classificado a partir dos valores de IMC preconizados pelo SISVAN7, sendo: baixo peso $I M C<18,5 \mathrm{Kg} / \mathrm{m}^{2}$; eutrofia $I M C>18,5$ e $<25,0 \mathrm{Kg} / \mathrm{m}^{2}$; sobrepeso $\mathrm{IMC} \geq 25,0$ e $\quad<30 \mathrm{Kg} / \mathrm{m}^{2}$; 
obesidade $\geq 30 \mathrm{Kg} / \mathrm{m}^{2}$. Em relação à circunferência abdominal (CA), a medida foi realizada com 0 participante em pé, utilizando-se uma fita métrica inflexível (Sanny® ES-2040) de precisão de 0,5cm. A fita circulou o participante no maior perímetro abdominal, entre a última costela e a crista ilíaca. A leitura foi feita no momento da expiração. Utilizaram-se os pontos de corte do SISVAN ${ }^{7}:<94 \mathrm{~cm}$ (sem risco para doenças cardiovasculares) e $>94 \mathrm{~cm}$ (risco elevado para doenças cardiovasculares).

Em relação ao período de sono, questionou-se: quantas horas os caminhoneiros dormem por noite, quantos dias dormem fora de casa por mês, se dormem após o almoço e o período de sono.

A análise dos dados foi realizada utilizando-se o software SPSS® (Statistical Package for the Social Sciences), versão 17.0. O nível de significância adotado foi de $5 \%(p \leq 0,05)$. As variáveis quantitativas foram descritas por média e desvio padrão e as variáveis qualitativas, por freqüências absolutas e relativas. Para comparar médias em dois grupos, o teste $t$-Student foi utilizado. Para mais de dois grupos, a análise de variância (ANOVA) one-way com post-hoc de Tukey foi aplicado. $\mathrm{Na}$ associação entre as variáveis categóricas, o teste qui-quadrado de Pearson ou exato de Fisher foram aplicados. Em caso de significância estatística, o teste dos resíduos ajustados foi utilizado. Para a associação entre as variáveis quantitativas ou ordinais, os testes da correlação linear de Pearson ou Spearman foram aplicados.

O estudo foi aprovado pelo Comitê de Ética em Pesquisa da Universidade Feevale de Novo Hamburgo, RS, sob protocolo número 4.05.03.11.1959.

\section{RESULTADOS}

Foram avaliados 100 caminhoneiros no município de Veranópolis/RS, com média de idade de 38,5 $\pm 10,2$ anos. Em relação à escolaridade, $52 \%$ relataram ter ensino fundamental incompleto, $24 \%$ ensino médio completo, $23 \%$ ensino fundamental completo e apenas $1 \%$ ensino superior completo. Quando questionados sobre a renda em salário mínimo ( $R \$ 545,00)$, 55\% ganham de 3-4 salários mínimos, $36 \%$ de 1-2 salários, 8\% de 5-6 salários mínimos e 1\% mais que 6 salários.
Quanto aos hábitos de vida, 67\% dos caminhoneiros citaram não praticar exercício físico. A ingestão de bebidas alcoólicas foi relatada por $60 \%$ deles. Observou-se que $85 \%$ não possuem o hábito de fumar. A maioria dos caminhoneiros (78\%) não utiliza medicamento para tratamento de patologias. Destes, $13 \%$ relataram possuir diagnóstico de hipertensão arterial, $7 \%$ de hipercolesterolemia, $5 \%$ de hipertrigliceridemia, e 3\% de diabete mellitus tipo 2 .

A média do IMC foi de $29,0 \pm 5,0 \mathrm{~kg} / \mathrm{m}^{2}$, sendo que $43 \%$ dos caminhoneiros classificaram-se em sobrepeso, $39 \%$ em obesidade e $18 \%$ em eutrofia. A média da CA foi de $100,9 \pm 14,0 \mathrm{~cm}$, com $69 \%$ da amostra apresentando risco elevado para o desenvolvimento de doenças cardiovasculares.

$\mathrm{Na}$ tabela 1, encontram-se descritos os hábitos de sono e auto-prescrição de medicamentos para não dormir. Observou-se que o número médio de horas de sono ao dia foi de $6,0 \pm 1,4 \mathrm{~h}$. Em relação à quantidade de dias que dormem fora de casa por mês, $51 \%$ dos caminhoneiros dormem de 1 a 5 dias, e 23\% não dormem nenhum dia fora de casa. Quanto à quantidade de horas de sono noturno, observou-se que $45 \%$ dos caminhoneiros dormem de $7-8$ horas e $40 \%$ dormem de 5-6 horas. O hábito de dormir após o almoço foi relatado por $25 \%$ dos caminhoneiros. Apenas $3 \%$ deles utilizam medicamento para não dormir, sendo consumido o "rebite".

Não houve diferença significativa entre as variáveis sociodemográficas e de hábitos de vida com o total de horas de sono/dia (tabela 2). Por outro lado, verificou-se diferença significativa nas variáveis colesterol total elevado e diabete mellitus. Entre os caminhoneiros com colesterol total elevado, houve mais horas de sono/dia em comparação com aqueles com colesterol total adequado. Para a DM ocorreu o inverso, ou seja, caminhoneiros diabéticos dormem menos do que os que não têm a doença (tabela 3).

Ao questionar sobre as práticas alimentares, foi verificado que a média de refeições por dia foi de $3,4 \pm 0,9$. A maioria deles (79\%) costuma tomar café da manhã: $41 \%$ em casa, $23 \%$ em restaurantes e $15 \%$ na caixa do caminhão. O lanche da manhã não é realizado por $79 \%$ dos caminhoneiros, e, entre aqueles que o 
TABELA 1 - Distribuição da amostra quanto ao padrão de sono.

\begin{tabular}{ll}
\hline Variáveis & $\mathbf{n = 1 0 0}$ \\
\hline Quantos dias dormem fora de casa/mês - $\mathbf{n}(\%)$ & \\
0 & 23,0 \\
$1-5$ & 51,0 \\
$6-10$ & 1,0 \\
$11-15$ & 4,0 \\
$16-20$ & 6,0 \\
$21-25$ & 13,0 \\
$26-30$ & 1,0 \\
$>30$ & 1,0 \\
Quantas horas dormem/noite - $\mathbf{n}(\%)$ & \\
$1-2$ & 1,0 \\
$3-4$ & 6,0 \\
$5-6$ & 40,0 \\
$7-8$ & 45,0 \\
$>8$ & 8,0 \\
Dorme após o almoço - $\mathbf{n}(\%)$ & 25,0 \\
Sim & 75,0 \\
Não & $6,0 \pm 1,4$ \\
Média de horas de sono/dia ( $\pm \mathrm{DP})$ & $3,0^{\star}$ \\
Utiliza medicamento para não dormir - \% & \\
\hline
\end{tabular}

*Todos utilizam rebite; \%=porcentagem; $\mathrm{n}=$ número; DP=desviopadrão.

realizam, $9 \%$ o fazem na caixa do caminhão, $7 \%$ em restaurantes e $5 \%$ em casa. Em relação ao almoço, $99 \%$ costumam almoçar, sendo $67 \%$ em restaurantes, $20 \%$ em casa e $12 \%$ na caixa do caminhão. O lanche da tarde não é realizado por $73 \%$ dos caminhoneiros; dos que o realizam, $12 \%$ o fazem na caixa do caminhão, $11 \%$ em restaurantes e $4 \%$ em casa. Em relação ao jantar, $98 \%$ deles dizem realizar esta refeição: $53 \%$ em casa, $26 \%$ em restaurantes e $19 \%$ na caixa do caminhão. Quanto à ceia, $91 \%$ dos caminhoneiros responderam que não realizam, sendo que, daqueles que a realizam, $8 \%$ fazem a ceia em casa e $1 \%$ na caixa do caminhão.

Houve associação significativa entre 0 total de dias que os caminhoneiros dormem fora de casa e o tipo de refeição. Caminhoneiros que dormem a semana inteira em casa apresentaram maior prevalência de realização do café da manhã, do almoço e do jantar em suas próprias residências. Os que dormem de 1-5 dias fora de casa apresentaram maior prevalência de realização do almoço e do jantar em restaurantes e de não realização do café da manhã. Aqueles que dormem mais de 5 dias fora de casa costumam realizar o café da manhã, o almoço e o jantar na caixa do caminhão (tabela 4).

Verificou-se associação significativa da faixa etária, do consumo de bebidas alcoólicas e da realização de lanche da tarde com dormir após o almoço (figura 1). Das associações com dormir após o almoço, houve significância estatística com a faixa etária $(p=0,044)$, etilismo $(p=0,020)$ e realização do lanche da tarde $(p=0,013)$. Indivíduos que dormem após o almoço são mais velhos ( $>50$ anos), referem tomar bebidas alcoólicas e ingerir lanche da tarde.

A figura 2 apresenta a associação significativa dos obesos com a presença de hipertensão $(p=0,035)$.

\section{DISCUSSÃo}

A faixa etária apresentada pelos caminhoneiros do estudo foi semelhante à apresentada por Lemos et al. ${ }^{8}$ em Campinas (SP) e em Belo Horizonte (MG), realizada com 209 caminhoneiros do sexo masculino entre $22 \mathrm{e}$ 62 anos de idade. Dentre os caminhoneiros avaliados, $52 \%$ relataram possuírem ensino fundamental incompleto, seguindo a tendência do estudo de Codarin et al. ${ }^{6}$, com $49,1 \%$ tendo a mesma escolaridade.

Em relação ao consumo de bebidas alcoólicas, a maioria $(60 \%)$ afirmou consumir algum tipo, corroborando com os dados de Ulhôa et al. ${ }^{9}$, que indicam que $65,5 \%$ dos motoristas de caminhão do estudo consumiam bebidas alcoólicas. Souza et al. ${ }^{10}$, ao avaliarem 406 caminhoneiros (200 de Portugal e 206 do Brasil), constataram que $73,5 \%$ dos caminhoneiros portugueses e $50,9 \%$ dos brasileiros faziam o uso de bebidas alcoólicas no seu dia a dia.

Conforme publicação de Domingos et al. ${ }^{11}$, o menor consumo alcoólico encontrado em seu estudo pelos brasileiros foi atribuído ao número de campanhas e à realização de fiscalização, mas, mesmo assim, observase que a quantidade de motoristas que ingerem bebidas alcoólicas nas estradas ainda é preocupante. Freqüentemente o consumo de álcool entre os motoristas ocorre nos horários de descanso e também durante as refeições como um meio de socialização. As bebidas são adquiridas em postos de gasolina $e$ restaurantes. Os dados desses estudos apontam que o consumo de bebidas alcoólicas ainda é um grave problema de saúde pública, pois motoristas nessas condições podem ocasionar sérios acidentes de trânsito ${ }^{11}$. Outro aspecto a observar é que, segundo o 
TABELA 2 - Avaliação das variáveis sociodemográficas e de hábitos de vida com o total de horas de sono.

\begin{tabular}{|c|c|c|}
\hline Variáveis & $\begin{array}{c}\text { Horas de sono } \\
\text { Média } \pm \text { DP }\end{array}$ & $\boldsymbol{P}$ \\
\hline Faixa etária & & 0,372 \\
\hline $21-30$ & $5,9 \pm 1,5$ & \\
\hline $31-40$ & $5,9 \pm 1,4$ & \\
\hline $41-50$ & $6,0 \pm 1,3$ & \\
\hline $51-60$ & $6,7 \pm 1,4$ & \\
\hline Nível de escolaridade & & 0,081 \\
\hline Fundamental incompleto/completo & $6,3 \pm 1,3$ & \\
\hline Ensino médio incompleto/completo & $5,6 \pm 1,5$ & \\
\hline Superior incompleto/completo & $5,9 \pm 1,4$ & \\
\hline Renda (salário mínimo) & & 0,416 \\
\hline $1-2$ & $6,3 \pm 1,3$ & \\
\hline $3-4$ & $5,9 \pm 1,5$ & \\
\hline$\geq 5$ & $6,2 \pm 1,5$ & \\
\hline Atividade Física & & 0,162 \\
\hline Sim & $5,8 \pm 1,5$ & \\
\hline Não & $6,2 \pm 1,4$ & \\
\hline Consome bebida alcoólica & & 0,723 \\
\hline $\operatorname{Sim}$ & $6,1 \pm 1,3$ & \\
\hline Não & $6,0 \pm 1,6$ & \\
\hline Fumante & & 0,952 \\
\hline Sim & $6,0 \pm 1,4$ & \\
\hline Não & $6,1 \pm 1,4$ & \\
\hline Usa medicação & & 0,355 \\
\hline $\operatorname{Sim}$ & $6,3 \pm 1,4$ & \\
\hline Não & $6,0 \pm 1,4$ & \\
\hline
\end{tabular}

Guia Alimentar para a população brasileira ${ }^{12}$, as bebidas alcoólicas contêm pouco ou nenhum nutriente, além de serem calóricas, contribuindo, assim, para o sobrepeso. As bebidas podem causar dependência e afetar as funções mentais, neurológicas e emocionais. Podem também provocar esquecimento e aumento do risco de demência.

Dentre os fatores ambientais pesquisados no estudo, destaca-se a falta de exercício físico pelos caminhoneiros (67\%). Esses dados vão ao encontro de Cavagioni et al. ${ }^{3}$, que demonstraram que $74 \%$ dos motoristas pesquisados não realizavam nenhum tipo de atividade física; e ao estudo de Masson et al. ${ }^{13}$, que obteve $77,1 \%$ de sedentários. Por outro lado, Codarin et al. ${ }^{6}$ encontraram apenas $54,2 \%$ de indivíduos sedentários. Comparando todos esses estudos, de um modo geral, observou-se que os caminhoneiros têm comportamento sedentário, em que a disponibilidade de tempo, fatores relacionados aos costumes da região onde residem e características individuais são as principais causas para não praticarem atividades físicas de qualquer tipo 6 .

A inadequação nos hábitos de sono pode causar consequências a curto e em longo prazo, dependendo do número de horas e da freqüência de sono. Em curto prazo, o efeito pode ser a redução da atenção e da concentração, a redução de produtividade e o aumento do número de acidentes. As consequências em um período maior de irregularidade do sono incluem aumento da morbidade e mortalidade em acidentes e o desenvolvimento de doenças, entre elas, insuficiência cardíaca, pressão arterial elevada, obesidade, diabetes tipo 2 (DM2), acidente vascular cerebral, perda de memória e depressão. Todavia, ainda existem controvérsias sobre as conseqüências relacionadas acima em se tratando da irregularidade do sono a longo prazo $^{14}$.

A média de horas de sono diário, neste estudo, se aproximou do encontrado por Souza et. al. ${ }^{15}$, que foi de $5,97 \pm 1,47$ ( 2 a $10 \mathrm{~h}$ ), sendo que $50,4 \%$ destes relataram dormir de 5 a 6 horas. Estudo de Guimarães et al. ${ }^{16}$ constatou que a maioria (89\%) dormia de 6 a 8 horas, resultado também compatível com o presente estudo.

Observou-se entre os resultados da pesquisa uma tendência à associação entre nível de escolaridade e o total de horas de sono, sendo que os indivíduos com 
TABELA 3 - Avaliação das variáveis de Doença crônica não transmissível e parâmetros antropométricos com o total de horas de sono.

\begin{tabular}{|c|c|c|}
\hline Variáveis & $\begin{array}{l}\text { Horas de sono } \\
\text { Média } \pm \text { DP }\end{array}$ & $P$ \\
\hline Doenças crônicas & & \\
\hline HAS & & 0,189 \\
\hline Sim & $6,5 \pm 1,5$ & \\
\hline Não & $6,0 \pm 1,4$ & \\
\hline CT elevado & & 0,029 \\
\hline Sim & $6,7 \pm 0,7$ & \\
\hline Não & $6,0 \pm 1,4$ & \\
\hline TG elevado & & 0,229 \\
\hline Sim & $6,6 \pm 0,9$ & \\
\hline Não & $6,0 \pm 1,4$ & \\
\hline$D M$ & & $<0,001$ \\
\hline Sim & $5,1 \pm 0,1$ & \\
\hline Não & $6,1 \pm 1,4$ & \\
\hline Classificação do IMC & & 0,666 \\
\hline Eutrofia $(18,5-24,9)$ & $5,9 \pm 1,2$ & \\
\hline Sobrepeso $(25,0-29,9)$ & $6,0 \pm 1,6$ & \\
\hline Obesidade $(\geq 30)$ & $6,2 \pm 1,3$ & \\
\hline Classificação da CA & & 0,351 \\
\hline Sem risco $(<94 \mathrm{~cm})$ & $5,8 \pm 1,5$ & \\
\hline Risco elevado $(\geq 94 \mathrm{~cm})$ & $6,1 \pm 1,4$ & \\
\hline
\end{tabular}

$\mathrm{DP}=$ desvio padrão; $\mathrm{p}=0,05$; HAS=hipertensão arterial sistêmica; $\mathrm{CT}=$ colesterol total; TG=triglicerídeos; $\mathrm{DM}=$ diabete mellitus; IMC= índice de massa corporal; $\mathrm{CA}=$ circunferência abdominal

baixa escolaridade dormem mais $(6,3 \pm 1,3)$ do que os com maior conhecimento $(5,7 \pm 1,5)$. Uma explicação para isso talvez esteja no fato de que os caminhoneiros com baixa escolaridade são os mais velhos. Assim, possivelmente, estes se cansem mais facilmente $e$ tenham hábitos diferentes dos motoristas mais jovens, dormindo por mais tempo. Não foram encontrados estudos científicos em caminhoneiros com essa associação.

Neri et al. ${ }^{17}$ apontam algumas condições de saúde e de trabalho de motoristas, indicando que a fadiga e a irritabilidade são as principais queixas entre eles, devido a horários a serem cumpridos e preocupações diárias decorrentes da profissão, consequentemente desenvolvendo estresse. O excesso de horas de trabalho também é um fator que está relacionado aos distúrbios do sono

Houve poucos relatos das DCNT neste estudo. Cabe ressaltar que estes dados foram obtidos a partir do relato dos avaliados sobre o diagnóstico médico para tal. Diferente do apresentado, Cavagioni et al. ${ }^{18}$ demonstraram prevalências de $37,2 \%$ de hipertensão arterial, $38,3 \%$ de triglicerídeos e $33 \%$ de colesterol total elevado. Observou-se, também, que quanto maior o IMC aumentavam os casos de hipertensão. O mesmo ocorreu com a glicemia. Quintana et al. ${ }^{19}$ apresentaram alguns fatores conhecidos para o desenvolvimento de hipertensão, tais como, traços de personalidade, depressão, ansiedade, genética e fatores ambientais, como a obesidade, inatividade física e excesso de consumo de sódio.

No presente estudo, observou-se relação das variáveis de DCNT, Diabete Mellitus (DM) e Colesterol Total (CT) elevado com total de horas de sono. Os indivíduos com colesterol elevado dormem mais se comparados com os que não possuem essa alteração. Cavagioni et al. ${ }^{3}$, em seu estudo com 258 motoristas na Rodovia BR-116, observaram que $33 \%$ deles possuíam níveis elevados de CT, porém, não houve associações com as horas de sono.

Outro dado observado foi o de que os caminhoneiros que apresentaram DM tipo 2 dormem menos do que os que não têm a doença. Conforme Lam et al. ${ }^{20}$, indivíduos que dormem menos de $5 \mathrm{~h}$ ou mais de $9 \mathrm{~h}$ estão mais propensos a desenvolver DM tipo 2. A influência da qualidade do sono no metabolismo da glicose ainda está sendo investigada. Alguns distúrbios do sono, como dificuldade de iniciar e manter o sono, foram associados com um maior risco para desenvolver diabetes. Assim, a duração e a qualidade mostraram-se significantes no controle glicêmico, ou seja, é importante na regulação da glicose, que envolve a resposta das células $\beta$ e a sensibilidade à insulina. Houve também associação com a obesidade. A gordura visceral produz grandes quantidades de citocinas pró-inflamatórias, que são importantes para o estresse oxidativo, para a adesão celular e, consequentemente, contribuem para 0 desenvolvimento da aterosclerose ${ }^{20}$.

A diminuição do tempo de sono altera a leptina e desencadeia um sinal no balanço energético, ou seja, na saciedade quando as necessidades calóricas são alcançadas. A leptina em circulação é causada devido às mudanças no balanço energético, resultado do aumento ou da diminuição de ingestão calórica; o jejum ou a perda de massa corporal ocasionam baixos níveis de leptina no sangue. A grelina é aumentada nos períodos de jejum, ocasionando a sensação de fome, a motilidade gastrointestinal e promovendo a deposição de gordura. Altos níveis desse hormônio pela manhã estão relacionados com a curta duração do sono ${ }^{5}$. 
TABELA 4 - Associação das práticas alimentares com o total de dias que dorme fora de casa.

\begin{tabular}{|c|c|c|c|c|}
\hline \multirow[b]{2}{*}{ Tipo e local da realização das refeições } & \multicolumn{3}{|c|}{ Total de dias que dorme fora de casa } & \multirow[b]{2}{*}{$P$} \\
\hline & $\begin{array}{l}\text { Zero } \\
\mathrm{n}(\%)\end{array}$ & $\begin{array}{c}1-5 \\
\mathrm{n}(\%)\end{array}$ & $\begin{array}{c}>5 \\
n(\%)\end{array}$ & \\
\hline Café da manhã & & & & $<0,001$ \\
\hline Não realiza & $5(21,7)$ & $15(29,4)^{*}$ & $1(3,8)$ & \\
\hline Em casa & $14(60,9)^{*}$ & $23(45,1)$ & $4(15,4)$ & \\
\hline Restaurante & $4(17,4)$ & $12(23,5)$ & $7(26,9)$ & \\
\hline Caixa caminhão & $0(0,0)$ & $1(2,0)$ & $14(53,8)^{*}$ & \\
\hline Lanche da manhã & & & & 0,643 \\
\hline Não realiza & $17(73,9)$ & $40(78,4)$ & $22(84,6)$ & \\
\hline Em casa & $1(4,3)$ & $4(7,8)$ & $0(0,0)$ & \\
\hline Restaurante & $3(13,0)$ & $3(5,9)$ & $1(3,8)$ & \\
\hline Caixa caminhão & $2(8,7)$ & $4(7,8)$ & $3(11,5)$ & \\
\hline Almoço & & & & $<0,001$ \\
\hline Não realiza & $0(0,0)$ & $1(2,0)$ & $0(0,0)$ & \\
\hline Em casa & $12(52,2)^{*}$ & $7(13,7)$ & $1(3,8)$ & \\
\hline Restaurante & $11(47,8)$ & $39(76,5)^{*}$ & $17(65,4)$ & \\
\hline Caixa caminhão & $0(0,0)$ & $4(7,8)$ & $8(30,8)^{*}$ & \\
\hline Lanche da tarde & & & & 0,192 \\
\hline Não realiza & $15(65,2)$ & $40(78,4)$ & $18(69,2)$ & \\
\hline Em casa & $1(4,3)$ & $3(5,9)$ & $0(0,0)$ & \\
\hline Restaurante & $5(21,7)$ & $4(7,8)$ & $2(7,7)$ & \\
\hline Caixa caminhão & $2(8,7)$ & $4(7,8)$ & $6(23,1)$ & \\
\hline Jantar & & & & $<0,001$ \\
\hline Não realiza & $0(0,0)$ & $1(2,0)$ & $1(3,8)$ & \\
\hline Em casa & $20(87,0)^{*}$ & $29(56,9)$ & $4(15,4)$ & \\
\hline Restaurante & $3(13,0)$ & $20(39,2)^{*}$ & $3(11,5)$ & \\
\hline Caixa caminhão & $0(0,0)$ & $1(2,0)$ & $18(69,2)^{*}$ & \\
\hline Ceia & & & & 0,102 \\
\hline Não realiza & $19(82,6)$ & $47(92,2)$ & $25(96,2)$ & \\
\hline Em casa & $4(17,4)$ & $4(7,8)$ & $0(0,0)$ & \\
\hline Caixa caminhão & $0(0,0)$ & $0(0,0)$ & $1(3,8)$ & \\
\hline
\end{tabular}

Outro dado importante é relativo aos efeitos do cortisol na transcrição do gene. A velocidade destes para a rota de síntese de glicose a partir de aminoácidos é induzida pelo cortisol. As alterações nas secreções que causam um estresse crônico resultam na influência do metabolismo energético, ou seja, ocorre diminuição da intolerância à glicose caso persistam altos níveis de cortisol $^{21}$.

Quanto ao IMC dos motoristas deste estudo, não houve associação significante com as horas de sono. No entanto, observou-se que $43 \%$ deles estavam com sobrepeso, $39 \%$ com obesidade e o restante em eutrofia, corroborando o estudo de Domingos et al. ${ }^{11}$, no qual $71,8 \%$ dos motoristas encontravam-se acima do peso. Masson et al ${ }^{13}$ também observaram $40,9 \%$ dos motoristas com sobrepeso, resultado próximo ao encontrado no presente estudo. Nenhum dos estudos mencionados acima, assim como nesta pesquisa, encontrou relação com IMC e período de sono. Quanto à circunferência da cintura $(\mathrm{CC})$, a média encontrada no presente estudo foi de $(100,9 \pm 14,0 \mathrm{~cm})$, compatível com o estudo de Cavagioni et al. ${ }^{18}$, em que a média foi de $(103,1 \pm 13,0 \mathrm{~cm})$.

Apesar de não encontrarmos associação entre o tempo de sono com IMC e CC, Crispim et al. ${ }^{5}$ apontam uma explicação para o excesso de peso dos motoristas. Os autores referem que, quando somado o excesso de peso com a redução do período de sono, existe uma ligação direta entre dois fatores endócrinos paralelos que são capazes de interferir na ingestão alimentar, sendo a diminuição do hormônio leptina e o aumento do hormônio grelina. A redução do tempo de sono afeta o apetite devido a esses dois hormônios participarem da homeostase, tanto da massa corporal como do sono. A leptina e a grelina alteradas geram um mecanismo capaz de alterar a ingestão alimentar elevar a desajustes nutricionais.

Quando avaliadas as práticas alimentares, as três refeições mais realizadas pelos motoristas foram o café da manhã, o almoço e o jantar. Isso pode ocorrer devido ao fato de estarem acostumados somente com as grandes refeições diárias, excluindo os lanches 


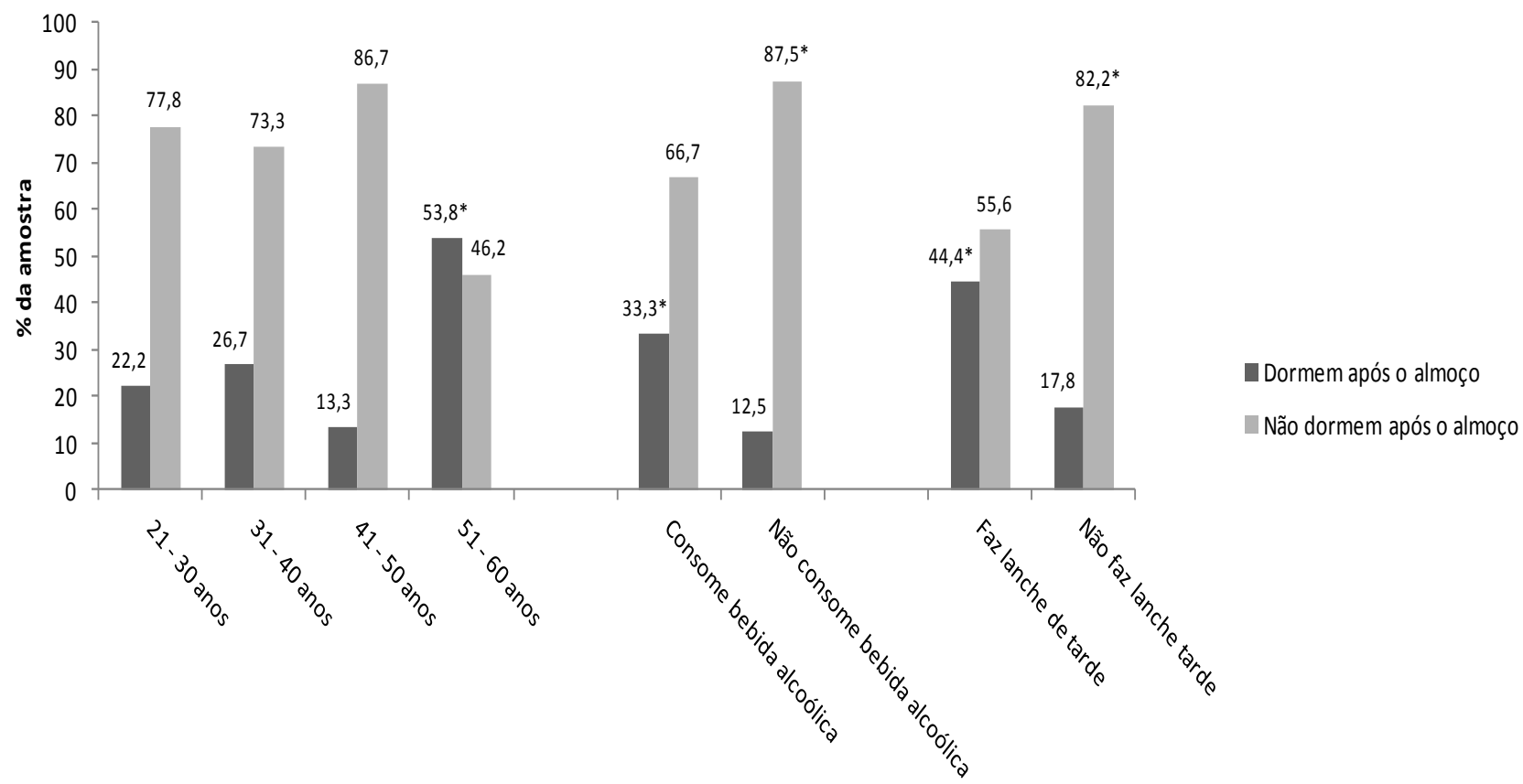

FIGURA 1 - Associação entre faixa etária, consumo de bebida alcoólica e realização de lanche da tarde com dormir após o almoço. *associação estatisticamente significativa pelo teste dos resíduos ajustados a $5 \%$ de significância.

intermediários. Quanto aos locais de realização das refeições, ficou evidente a residência como o local mais prevalente quando eles não dormem nenhum dia fora de casa, e restaurantes quando estão trabalhando. O Guia Alimentar para a população brasileira ${ }^{12}$ orienta que é importante que todas as pessoas realizem pelos menos três refeições diárias (café da manhã, almoço e jantar), intercaladas por pequenos lanches. A inclusão de lanches intermediários na rotina diária dos caminhoneiros é de suma importância nutricional, pois auxiliam o controle da ingestão de grande volume de alimentos nas refeições maiores. As alternativas cabíveis para a realização destas refeições seriam: conseguir parar de tempo em tempo em locais como lanchonetes ou postos de combustíveis para sua realização, ou levar os lanches prontos de casa. Normalmente as refeições realizadas fora de casa (em restaurantes), e não o volume das refeições pode ser mais preocupante, pois os tipos de nutrientes, principalmente carboidratos, estão presentes na maioria das refeições e são os que aumentamos níveis de grelina $^{22}$.

Observou-se associação entre dormir após o almoço com faixa etária, consumo de álcool e realizar lanche da tarde entre os homens mais velhos. Esses dados podem ser atribuídos ao fato de que, com a idade, talvez esses caminhoneiros tenham menos compromissos com o trabalho, tenham mais disponibilidade e realizem viagens mais curtas, porém, não encontramos estudos comparativos com essa associação.

Com base nessas considerações, os resultados deste estudo sugerem que a maioria dos caminhoneiros estudados apresenta estado nutricional desfavorável, com prevalência de sobrepeso e obesidade e em risco elevado para 0 desenvolvimento de doenças cardiovasculares. O total de horas de sono diário teve relação com as DCNT (colesterol total elevado e diabete mellitus). Em geral, os caminhoneiros realizam três refeições diárias, sendo que, aqueles que dormem de 1 a 5 dias fora de casa fazem apenas duas. $O$ hábito de praticar exercícios físicos e o tabagismo não fazem parte da rotina dos caminhoneiros, por outro lado, a ingestão de bebidas alcoólicas foi bastante prevalente. Apesar das limitações deste estudo, como a dificuldade para obtenção de dados subjetivos em relação às DCNT, os resultados obtidos podem servir como base para o planejamento de intervenções de educação em saúde, apoiadas na motivação dos caminhoneiros às medidas de prevenção primária de doenças cardiovasculares, tais como hábitos alimentares, prática 

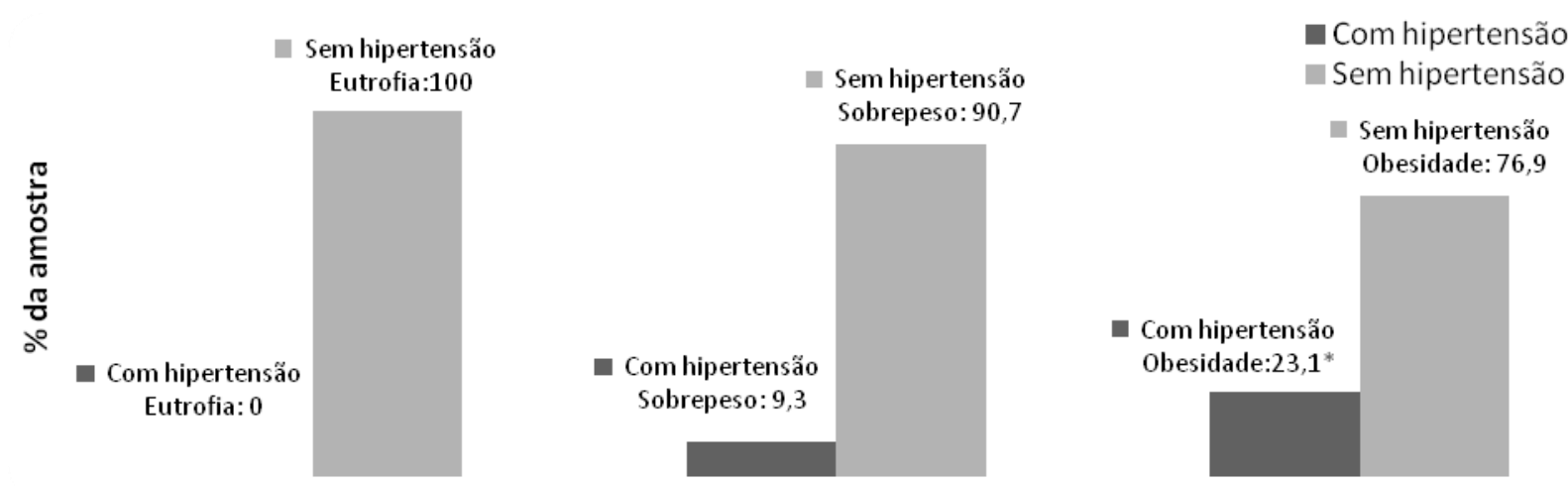

FIGURA 2 - Prevalência de hipertensão, conforme classificação do IMC. *associação estatisticamente significativa pelo teste dos resíduos ajustados a 5\% de significância.

de atividades físicas regulares e eliminação do etilismo. A associação de práticas alimentares saudáveis com atividade física e adequado tempo de sono podem ajudar no controle do excesso de peso e minimizar o aparecimento de doenças. Entretanto, torna-se importante a realização de outros estudos para esclarecer e elucidar a relação do sono com o estado nutricional e os hábitos de vida em caminhoneiros.

\section{REFERÊNCIAS}

1. Ornellas TCF, Monteiro MI. Aspectos históricos, culturais e sociais do trabalho. Rev Bras Enferm. 2006; 59(4):552-5.

2. Brasil. Ministério de Trabalho e Emprego. Classificação brasileira de ocupações. Brasília (DF): Ministério de Trabalho e Emprego; 2002. Disponível em: http://www.mtecbo.gov.br/busca/descricao.asp?codigo=78 25.

3. Cavagioni LC, Bensenõr IM, Halpern A, Pierin AMG. Síndrome metabólica em motoristas profissionais de transporte de cargas da rodovia BR-116 no trecho Paulista-Régis Bittencourt. Arq Bras Endocrinol Metab. 2008; 52(6):1015-23.

4. Nascimento EC, Nascimento E, Silva JP. Uso de álcool e anfetaminas entre caminhoneiros de estrada. Rev Saúde Pública. 2007; 41(2):290-3.

5. Crispim CA, Zalcman I, Dáttilo M, Padilha HG, Tufik $S$, Mello MT. Relação entre sono e obesidade: uma revisão da literatura. Arq Bras Endocrinol Metab. 2007; 51(7):1041-9.

6. Codarin MAF, Moulatlet EM, Nehme P, Ulhôa M, Moreno CRC. Associação entre prática de atividade física, escolaridade e perfil alimentar de motoristas de caminhão. Saúde Soc. 2010; 19(2):418-28.

7. Brasil. Ministério da Saúde. Orientações para a coleta e análise de dados antropométricos em serviços de saúde: Norma Técnica do Sistema de Vigilância Alimentar e Nutricional- SISVAN. Brasília (DF): Ministério da Saúde; 2011.

8. Lemos LC, Marqueze EC, Sachi F, Filho GL, Moreno CRC. Síndrome da apnéia obstrutiva do sono em motoristas de caminhão. J Bras Pneumol. 2009; 35(6):500-6.

9. Ulhôa MA, Marqueze EC, Lemos LC, Silva LG, Silva AA, Nehme P, Fischer FM, Moreno CRC. Distúrbios psíquicos menores e condições de trabalho em motoristas de caminhão. Rev Saúde Pública. 2010; 44(6):1130-6.

10. Souza JC, Paiva T, Reimão R. Sono, qualidade de vida e acidentes em caminhoneiros brasileiros e portugueses. Psicol Estud. 2008; 13(3):429-36.

11. Domingos JBC, Jora NP, Carvalho AMP, Pillon SC. Consumo de álcool, sobrepeso e obesidade entre caminhoneiros. Rev Enferm. 2010; 18(3):377-82.

12. Brasil. Ministério da Saúde. Guia Alimentar para a população brasileira: promovendo a alimentação saudável. Brasília (DF): Ministério da Saúde; 2008. Serie A. Normas e Manuais Técnicos.

13. Masson VA, Monteiro MI. Estilo de vida, aspectos de saúde e trabalho de motoristas de caminhão. Rev Bras Enferm. 2010; 63(4):533-40.

14. Chokroverty S. Overview of sleep \& sleep disorders. Indian J Med Res.2010; 131:126-40.

15. Souza JC, Paiva T, Reimão R. Sleep habits, sleepiness and accidents among truck drivers. Arq Neuropsiquiatr. 2005; 63(4):925-30.

16. Guimarães MASV, Silva MAA. Relação entre sono e voz: percepção de indivíduos adultos disfônicos e não disfônicos. Distúrb Comum. 2007; 19(1):93-102.

17. Neri M, Soares WL, Soares C. Condições de saúde no setor de transporte rodoviário de cargas e de passageiros: um estudo baseado na Pesquisa Nacional por Amostra de Domicílios. Cad Saúde Pública. 2005; 21(4):1107-23.

18. Cavagioni LC, Pierin AMG. Hipertensão arterial e obesidade em motoristas profissionais de transporte de cargas. Acta Paul Enferm. 2010; 23(4):455-60.

19. Quintana JF. A relação entre hipertensão com outros fatores de risco para doenças cardiovasculares e tratamento pela psicoterapia cognitivo comportamental. Rev SBPH. 2011;14(1):3-17.

20. Lam JCM, Ip MS. Sleep \& the metabolic syndrome. Indian J Med Res. 2010; 131:206-16.

21. Taheri S. A ligação entre a duração do sono curto e obesidade: devemos recomendar mais sono para prevenir a obesidade. Arch Dis Child.2006; 91(11):881-4.

22. Júnior AVV, Gomes CF, Allgayer N, Lara GM. A grelina e sua contribuição para obesidade e diabetes mellitus tipo 2 . Rev Conhec 2012; 4(2):1-8.

\section{Endereço para correspondência}

Patrícia De Paris

Rua Carlos Dreher Neto n० 1680

Bento Gonçalves/RS - CEP 95700-000

Telefone: +55 5496124775

E-mail: patideparis@hotmail.com 